\title{
26 Research Soure \\ Effectiveness of Antibiotic Treatment in Children with Lyme Neuroborreliosis - A Retrospective Study
}

\section{Sigurdur Arnason}

Department of Clinical Science, Intervention and Technology - CLINTEC, Karolinska Institutet, Stockholm

\section{Barbro H Skogman ( $\nabla$ barbro.hedinskogman@regiondalarna.se )}

Center for Clinical Research Dalarna - Uppsala University, Region Dalarna

\section{Research Article}

Keywords: Lyme neuroboreliosis (LNB), neurological manifestations, antibiotic treatment, doxycycline p.o.

Posted Date: December 17th, 2021

DOI: https://doi.org/10.21203/rs.3.rs-1137228/v1

License: (c) (1) This work is licensed under a Creative Commons Attribution 4.0 International License. Read Full License 


\section{Abstract}

\section{Background}

Lyme neuroboreliosis (LNB) is a tick-borne infection caused by the spirochete Borrelia burgdorferi sensu lato complex with various neurological manifestations. The recommended treatment for LNB in Swedish children has been ceftriaxone i.v. 50-100 mg/kg x 1 (< 8 years of age) or doxycycline p.o. $4 \mathrm{mg} / \mathrm{kg} \mathrm{x} 1$ ( $\geq$ 8 years of age) for 10-14 days. Studies on adult LNB patients have shown equal efficacy for ceftriaxone i.v. and doxycycline p.o., but no such studies have been performed on pediatric LNB patients. The aim of this study is to retrospectively evaluate clinical outcome in children with LNB who have received ceftriaxone i.v. as compared to doxycycline p.o.

\section{Results}

Clinical and laboratory data from three previously performed prospective studies on children with LNB (three cohorts, 1998-2014) were collected and retrospectively analyzed. A total of 321 Swedish children (1-19 years of age), who had received antibiotic treatment for LNB, were included. Clinical outcome at the 2-month follow-up (recovery/non-recovery) was evaluated, using $\mathrm{Chi}^{2}$ test and logistic multivariate regression analysis.

Out of 321 LNB patients, 194 children (60\%) had received ceftriaxone i.v. and 127 children (40\%) had received doxycycline p.o.. When comparing recovery/non-recovery between treatment groups, no difference in clinical outcome was found $(p=0,217)$. Results did not change when incorporating relevant clinical and laboratory data into the logistic multivariate regression analysis.

\section{Conclusion}

In this large retrospective study, no difference in clinical outcome (recovery/non-recovery) was found, independent of age, when comparing children who received ceftriaxone i.v. with children who received doxycycline p.o., supporting an equal effectiveness for treatment of pediatric LNB patients. However, future randomized comparative treatment studies with non-inferiority design are warranted for evaluation of efficacy and safety of antibiotic treatment in pediatric LNB patients.

\section{Background}

Lyme borreliosis (LB) is the most common tick-borne infection in the northern hemisphere. The infection is caused by the spirochaete Borrelia $(B$.) burgdorferi sensu lato complex $(1,2)$. The complex consists of 11 different species where the major human pathogens in Europe are B. afzeli, B. garini and B. burgdoferi sensu stricto (1-3). When the spirochetes emerge from the skin into the central nervous system (CNS), symptoms of subacute meningitis and/or cranial or peripheral nerve impairment may occur, resulting in

Lyme neuroborreliosis (LNB) (4). The most common manifestation of LNB in children is facial nerve palsy followed by symptoms of subacute meningitis (fever, headache, neck pain, neck stiffness) (5-7). 
Unspecific symptoms such as loss of appetite, change of mood or fatigue may sometimes be present in younger children (8). Clinical outcome is genereally favourable, but persistent symptoms may occur and affect daily life (9). The results of a lumbar puncture, in addition to clinical symptoms attributable to LNB, are required to determine the diagnosis of LNB (10). In the cerebrospinal fluid (CSF), pleocytosis (>5 $\mathrm{x}$ $10^{6} / \mathrm{L}$ white cells with a mononuclear cell dominance) and intrathecal production of specific anti-Borreliaantibodies are needed to confirm the LNB diagnosis (10).

In Sweden, the recommended treatment for LNB has been ceftriaxone i.v. $50-100 \mathrm{mg} / \mathrm{kg} \times 1$ (children < 8 years of age) or doxycycline p.o $4 \mathrm{mg} / \mathrm{kg} \times 1$ (children $\geq 8$ years of age) for 10-14 days, according to national guidelines. In latter years, the safety of tetracyclines has been in focus, mainly due to concerns of adverse effects such as dental staining and enamel hypoplasia in younger children. However, doxycycline has a lower calcium-binding capacity than previous generations of tetracyclines, and recent studies have shown that doxycycline is safe for children younger than 8 years of age (11-13). Furthermore, doxycycline p.o., contrary to ceftriaxone i.v., is safe, inexpensive, easy for parents to administer and hospitalized care is not needed (14). Extensive use of cephalosporins causes an overall increase in antibiotic resistance (mainly by affecting the intestinal microbial flora with an increased risk of Clostridium difficile infections) and it is of outmost importance to limit the overall use of cephalosporins in the healthcare system (15).

In Norway, a non-inferiority trial was performed in 2007 on adult LNB patients. Participants $(n=102)$ were randomly allocated to receive ceftriaxone i.v. $2 \mathrm{~g} \times 1$ or doxycycline p.o. $200 \mathrm{mg} \times 1$ for 14 days (16). After 4 months, patients were evaluated for clinical outcome by using a composite clinical score (range 0-64, 0 $=$ complete recovery and $64=$ no recovery). In the doxycycline group, 28 out of 54 (52\%) patients were completely recovered and in the ceftriaxone group, 16 out of $48(33 \%)$ patients were completely recovered (16). The study showed that doxycycline p.o. had an efficacy equal to ceftriaxone i.v. for treatment of LNB in adult patients (16).

In 2016 a systematic review was conducted on efficacy and safety of pharmacological treatments for LNB in children (17). Two randomized-control trials and four non-randomized studies on treatment of LNB in patients younger than 18 years of age were included (17). The authors conclude that there were no differences between the two strategies of antibiotic treatment (doxycycline p.o. versus ceftriaxone i.v.) in children with LNB, but the quality of evidence (GRADE) of the included studies was deemed very low. In addition, no evidence was found to support prolonged antibiotic treatment (17). In summary, there is still an important gap in knowledge concerning the most efficient strategy for antibiotic treatment in pediatric LNB patients.

The aim of this study is to retrospectively evaluate clinical outcome in children who have received ceftriaxone i.v. as compared to children who have receive doxycycline p.o. as antibiotic treatment for LNB.

\section{Material And Methods}




\section{Design}

Clinical and laboratory data from three previously performed prospective studies $(6,18,19)$ were collected and analyzed together in this retrospective study. The study periods were 1998-2001, 20002005, and 2010-2014 respectively. Data from these three studies will be referred to as Cohort 1, 2 and 3 (Table 1). The three cohorts together represent a large portion of pediatric LNB patients at seven pediatric departments in central and southeast Sweden, all of whom have received antibiotic treatment for LNB according to national guidelines.

\section{Patient sample and follow-up questionnaire}

The patient sample contained information about age, gender, symptoms, duration of neurological symptoms, diagnosis (Definite LNB or Possible LNB), antibiotic treatment (ceftriaxone i.v. or doxycycline p.o.; dose and duration of treatment), pleocytosis in CSF, intrathecally produced specific anti-Borrelia antibodies, and clinical outcome at 2-month follow-up (recovery/non-recovery). A total of 321 children between 1-19 years of age were included in the study. Patients were classified as Definite LNB $(n=228)$ or Possible LNB ( $n=92)$. The diagnosis of Definite LNB was defined according to the European case definition, by clinical symptoms attributable to LNB, mononuclear pleocytosis in CSF and intrathecally produced specific anti-Borrelia antibodies (10). The diagnosis of Possible LNB was defined by clinical symptoms attributable to LNB, mononuclear pleocytosis in CSF, absence of intrathecally produced specific anti-Borrelia antibodies, response to antibiotic treatment and no signs of other disease. All Definite LNB and Possible LNB patients received and were clinically improved on antibiotic treatment and are considered as LNB patients. Characteristics of LNB patients are shown in Table 1 and Figure 1.

At the 2-month follow-up, a structured questionnaire was used to document self/parent-reported persistent symptoms and a clinical examination was performed, including the House-Brackmann facial nerve grading scale (a physician-assessed six-point scale to evaluate facial nerve impairment) (20). Based on this information, LNB patients were defined as being recovered/not-recovered.

\section{Statistics}

Statistical analyses were performed in IBM SPSS Statistics, version 26 (IBM Corporation, USA)When comparing continuous data between groups, the Mann-Whitney $U$ test analysis was used. For noncontinuous data the $\mathrm{Chi}^{2}$ test analysis was used. A logistic multivariate regression analysis (generalized linear model), was used for all relevant clinical and laboratory variables with the dependent variable clinical outcome at the 2-month follow-up (recovery/non-recovery). A total of 317 participants were included in the logistic multivariate regression analysis. A p-value of $<0.05$ was considered significant.

\section{Ethics}

The three previous prospective studies were approved by the Regional Ethics Committee in Linköping (Dnr 98103 and Dnr 02-159) and Uppsala (Dnr 2010/106), Sweden. Every child was assigned a specific study-ID. Written informed consent was received from parents/guardians. 


\section{Results}

Out of 321pediatric LNB patients, 194 children (60\%) received ceftriaxone i.v. and 127 children (40\%) received doxycycline p.o. (Figure 2, Table 2). However, four patients had received both ceftriaxone i.v. and doxycycline p.o. during the treatment for LNB. Three of these patients were included as part of the ceftriaxone group, since the majority of their treatment was given intravenously. The fourth patient had received only one day of ceftriaxone i.v. followed by 13 days of doxycycline p.o. and was consequently included in the doxycycline group. In 38 LNB cases, the pediatrician had not followed the age recommendation and had chosen ceftriaxone i.v. instead of doxycycline p.o. for LNB treatment, even though the child was older than 8 years of age. Furthermore, one girl had received doxycycline, even though she was 4 years of age.

In the ceftriaxone group, 149 patients (77\%) were classified as Definite LNB and 45 patients (23\%) as Possible LNB. In the doxycycline group, 79 patients (62\%) were classified as Definite LNB and 48 patients $(38 \%)$ as Possible LNB (Table 2). Among patients in the ceftriaxone group, 155 out of 194 (80\%), were defined as having a complete recovery and 39 patients $(20 \%)$ were defined as having an incomplete recovery at the 2-month follow-up (Figure 2). In the doxycycline group, 94 patients (74\%) were completely recovered and 33 patients (26\%) were defined as having incomplete recovery (Figure 2 ). The most commonly reported persistent signs or symptoms at the 2-month follow-up, in both groups, were facial nerve palsy, headache and fatigue. Clinical and laboratory characteristics on admission and at follow-up are shown in Table 2.

There was no significant difference in clinical outcome at the 2-month follow-up (recovery/non-recovery) when comparing children treated for LNB with either ceftriaxone i.v. or doxycycline p.o. $(p=0,217)$. When relevant clinical and laboratory data on admission were included and analyzed in a logistic multivariate regression analysis, there was still no significant association between antibiotic treatment and clinical outcome (OR 1.05 with 95\%; Cl 0.51 - 2.17) (Table 3). Additionally, there were no significant associations between age, gender, known tick bite, erythema migrans, headache, fatigue or pleocytosis on admission and clinical outcome at the 2-month follow-up (Table 3). However, facial nerve palsy (OR 2.72 with $95 \% \mathrm{Cl}$ 1.29 - 5.79) and fever (OR 2.36 with 95\% Cl 1.24 - 4.50) were associated with poorer clinical outcome (non-recovery), whereas occurrence of anti-Borrelia-antibodies in CSF (i.e. patients classified as Definite LNB) was associated to better clinical outcome (recovery) (OR 0.41 with $95 \% \mathrm{Cl} 0.21-0.80$ ) (Table 3).

\section{Discussion}

In this large retrospective study on pediatric LNB patients, we have shown that there was no difference between children, independent of age, who had received ceftriaxone i.v. and those who had received doxycycline p.o. when comparing clinical outcome (recovery/non-recovery). Our results are in line with previous studies (17), supporting the hypothesis that doxycycline p.o. is as effective as ceftriaxone i.v. for treatment of LNB. However, the efficacy and safety of the two different treatment strategies could not 
fully be evaluated in our study, since it was not a randomized comparative study, and unknown confounding factors may have influenced our results.

One strength of our retrospective study was that results are based on data from a relatively larger patient sample $(n=321)$ including three previous prospective cohorts. Patients are well characterized and considered representative of Swedish pediatric LNB patients, and all participating children were clinically followed-up at 2 months, in all three cohorts. The follow-up visits were congruent and well executed by physicians at each pediatric department, including a clinical examination and a pre-defined structured questionnaire for self/parent-reported persistent symptoms. Patients were defined as being recovered/not-recovered based on findings from the examination and answers from the questionnaires. Unfortunately, for the assessment of clinical outcome, no clinical composite score nor validated questionnaire was used at the follow-up visits, which is a weakness of the study. However, we believe that the overall clinical evaluation of each patient, by pediatricians at the 2-month follow-up visit, was correct and sufficient to determine if the patient was recovered/not-recovered.

An additional limitation of the study is that we did not have precise data on the duration of antibiotic treatment, since children could have received a course of antibiotic treatment varying from 10 to14 days. Therefore, analysis of the association between treatment duration and clinical outcome was not feasible.

The duration of antibiotic treatment in children with early LNB has been under debate (21) and treatment for 10-30 days has been suggested $(17,22)$. Recently published evidence-based guidelines from Germany have determined a recommendation of 14 days of doxycycline, ceftriaxone, cefotaxime or Penicillin G i.v. (21).

The age of the children and the choice of antibiotic treatment was not always congruent with Swedish guidelines in our study. Thirtyeight $(n=38)$ children had received ceftriaxone i.v. even though they were $\geq$ 8 years of age.These patients could possibly have had a more severe LNB on admission than other children. However, with the regression analysis, including age, symptoms on admission and antibiotic treatment, this should not have influenced our results on clinical outcome.

The most common persistent symptom at the 2-month follow-up in our study was facial nerve palsy (16\%). Results were similar in both diagnostic groups (Definite LNB and Possible LNB) and in both treatment groups (ceftriaxone i.v. and doxycycline p.o.). Results are in line with previous studies $(9,23)$. Facial nerve palsy on admission was also one of the major clinical manifestations associated with a higher risk of non-recovery in our logistic multivariate regression analysis. This result is not surprising, and in line with earlier studies, where the validated House-Brackmann grading scale has also been used to evaluate clinical outcome $(23,24)$. However, the manifestation of fever on admission and its association with poorer clinical outcome was more surprising. This association could possibly be understood, as the fever itself being a sign of strong immunological activity in CSF in LNB, and the inflammation could negatively influence both the ability of clearing symptoms and clinical outcome (25). Anti-Borrelia antibodies in CSF were, contrary to facial nerve palsy and fever, associated with a better clinical outcome, possibly because of a faster and more determined decision for the start of treatment by 
the pediatricians in charge, which could have been beneficial for clinical recovery (9). Admittedly, the discussion about these associations is somewhat speculative and in some measure difficult to explain from a pathophysiological point of view, and should be interpreted with caution.

\section{Conclusion}

In this large retrospective study, no difference in clinical outcome (recovery/non-recovery) was found, independent of age, when comparing children who received ceftriaxone i.v. with children who received doxycycline p.o., supporting an equal effectiveness for treatment of pediatric LNB patients. However, future randomized comparative treatment studies with non-inferiority design are warranted for evaluation of efficacy and safety of antibiotic treatment in pediatric LNB patients.

\section{Abbreviations}

$\mathrm{Cl}$ - Confidence interval

CNS- Central nervous system

CSF - Cerebrospinal fluid

i.v. - intravenous

LB - Lyme borreliosis

LNB- Lyme neuroborreliosis

p.o. - peroral

\section{Declarations}

\section{Ethics approval and consent to participate}

All procedures performed in this study involving human participants (children) were in accordance with the ethical standards of the institutional and/or national research committee and with the $1964 \mathrm{Helsinki}$ declaration and its later amendments or comparable ethical standards. Approval of the study was obtained from the Regional Ethics Committee in Linköping (Dnr 98103 and Dnr 02-159) and Uppsala (Dnr 2010/106), Sweden. Every child was assigned a specific study-ID. Written informed consent was received from parents/guardians.

\section{Consent to publish}

Not applicable 
The dataset used and/or analyzed during the current study are available from the corresponding author on reasonable request.

\section{Competing interest}

The authors declare that they have no financial or non-financial competing interest.

\section{Funding}

Financial support was received from the Regional Research Council Uppsala-Örebro (RFR-226161, RFR462701), the Center for Clinical Research Dalarna - Uppsala University (CKFUU-105141, CKFUU-374651, CKFUU-566761), the Swedish Society of Medicine (SLS-498901, SLS-93191).

\section{Author's contribution}

BHS contributed with conception and design of the study, acquisition and analysis of data and drafting of the manuscript. SA contributed with analysis of data, drafting the article and revising the manuscript critically. Both authors approved the final version of the manuscript.

\section{Acknowledgement:}

Special thanks to Matilda Karlsson for work with data preparation and analysis, as part of her studies for Master of Science (MSc) at the Faculty of Medical and Health Sciences, Örebro University, Sweden

\section{References}

1. Stanek G, Wormser GP, Gray J, Strle F. Lyme borreliosis. Lancet. 2012;379(9814):461-73.

2. Marques AR, Strle F, Wormser GP. Comparison of Lyme Disease in the United States and Europe. Emerg Infect Dis. 2021;27(8):2017-24.

3. Steere AC, Strle F, Wormser GP, Hu LT, Branda JA, Hovius JW, et al. Lyme borreliosis. Nature reviews Disease primers. 2016;2:16090.

4. Oschmann P, Dorndorf W, Hornig C, Schafer C, Wellensiek HJ, Pflughaupt KW. Stages and syndromes of neuroborreliosis. J Neurol. 1998;245(5):262-72.

5. Sodermark L, Sigurdsson V, Nas W, Wall P, Trollfors B. Neuroborreliosis in Swedish Children: A Population-based Study on Incidence and Clinical Characteristics. Pediatr Infect Dis J. 2017;36(11):1052-6.

6. Skogman BH, Croner S, Nordwall M, Eknefelt M, Ernerudh J, Forsberg P. Lyme neuroborreliosis in children: a prospective study of clinical features, prognosis, and outcome. Pediatr Infect Dis J. 2008;27(12):1089-94. 
7. Tuerlinckx D, Glupczynski Y. Lyme neuroborreliosis in children. Expert Rev Anti Infect Ther. 2010;8(4):455-63.

8. Broekhuijsen-van Henten DM, Braun KP, Wolfs TF. Clinical presentation of childhood neuroborreliosis; neurological examination may be normal. Arch Dis Child. 2010;95(11):910-4.

9. Skogman BH, Glimaker K, Nordwall M, Vrethem M, Odkvist L, Forsberg P. Long-term clinical outcome after Lyme neuroborreliosis in childhood. Pediatrics. 2012;130(2):262-9.

10. Mygland A, Ljostad U, Fingerle V, Rupprecht T, Schmutzhard E, Steiner I, et al. EFNS guidelines on the diagnosis and management of European Lyme neuroborreliosis. Eur J Neurol. 2010;17(1):8-16, e1-4.

11. Todd SR, Dahlgren FS, Traeger MS, Beltran-Aguilar ED, Marianos DW, Hamilton C, et al. No visible dental staining in children treated with doxycycline for suspected Rocky Mountain Spotted Fever. J Pediatr. 2015;166(5):1246-51.

12. Poyhonen H, Nurmi M, Peltola V, Alaluusua S, Ruuskanen O, Lahdesmaki T. Dental staining after doxycycline use in children. J Antimicrob Chemother. 2017;72(10):2887-90.

13. Lopez SMC, Campfield BT, Nowalk AJ. Oral Management for Pediatric Lyme Meningitis. J Pediatric Infect Dis Soc. 2019;8(3):272-5.

14. Cross R, Ling C, Day NP, McGready R, Paris DH. Revisiting doxycycline in pregnancy and early childhood-time to rebuild its reputation? Expert Opin Drug Saf. 2016;15(3):367-82.

15. Karp J, Edman-Waller J, Toepfer M, Lundqvist A, Jacobsson G. Clostridioides difficile incidence related to in-hospital cephalosporin use: a tale of two highly comparable hospitals. J Antimicrob Chemother. 2019;74(1):182-9.

16. Ljostad U, Skogvoll E, Eikeland R, Midgard R, Skarpaas T, Berg A, et al. Oral doxycycline versus intravenous ceftriaxone for European Lyme neuroborreliosis: a multicentre, non-inferiority, doubleblind, randomised trial. Lancet Neurol. 2008;7(8):690-5.

17. Dersch R, Hottenrott T, Schmidt S, Sommer H, Huppertz HI, Rauer S, et al. Efficacy and safety of pharmacological treatments for Lyme neuroborreliosis in children: a systematic review. BMC Neurol. 2016;16(1):189.

18. Skogman BH, Croner S, Forsberg P, Ernerudh J, Lahdenne P, Sillanpaa H, et al. Improved laboratory diagnostics of Lyme neuroborreliosis in children by detection of antibodies to new antigens in cerebrospinal fluid. Pediatr Infect Dis J. 2008;27(7):605-12.

19. Backman K, Skogman BH. Occurrence of erythema migrans in children with Lyme neuroborreliosis and the association with clinical characteristics and outcome - a prospective cohort study. BMC Pediatr. 2018;18(1):189.

20. House JW, Brackmann DE. Facial nerve grading system. Otolaryngol Head Neck Surg. 1985;93(2):146-7.

21. Rauer S, Kastenbauer S, Hofmann H, Fingerle V, Huppertz HI, Hunfeld KP, et al. Guidelines for diagnosis and treatment in neurology - Lyme neuroborreliosis. Ger Med Sci. 2020;18:Doc03. 
22. Thorstrand C, Belfrage E, Bennet R, Malmborg P, Eriksson M. Successful treatment of neuroborreliosis with ten day regimens. Pediatr Infect Dis J. 2002;21(12):1142-5.

23. Arnason S, Hultcrantz M, Nilsson A, Laestadius A. Peripheral facial nerve palsy in children in a Borrelia high-endemic area, a retrospective follow-up study. Acta Paediatr. 2019.

24. Skogman BH, Croner S, Odkvist L. Acute facial palsy in children--a 2-year follow-up study with focus on Lyme neuroborreliosis. Int J Pediatr Otorhinolaryngol. 2003;67(6):597-602.

25. Skogman BH, Lager M, Brudin L, Jenmalm MC, Tjernberg I, Henningsson AJ. Cytokines and chemokines in cerebrospinal fluid in relation to diagnosis, clinical presentation and recovery in children being evaluated for Lyme neuroborreliosis. Ticks and tick-borne diseases. 2020;11(3):101390.

\section{Tables}

Table 1. Age, gender, known tick bite and erythema migrans in the three cohorts of children with Lyme neuroborreliosis

\begin{tabular}{lrrr} 
& Cohort 1 & Cohort 2 & Cohort 3 \\
\hline & $(\mathrm{n}=142)$ & $(\mathrm{n}=77)$ & $(\mathrm{n}=102)$ \\
\hline Age, median (range) & $10(2-19)$ & $6(1-18)$ & $7(2-15)$ \\
\hline Gender & & & \\
\hline \multicolumn{1}{c}{ Female, $\mathrm{n}(\%)$} & $71(50)$ & $30(39)$ & $45(44)$ \\
\hline \multicolumn{1}{c}{ Male, $\mathrm{n}(\%)$} & $71(50)$ & $47(61)$ & $57(56)$ \\
\hline Known tick bite, $\mathbf{n}(\%)$ & $82(58)$ & $42(55)$ & $59(58)$ \\
\hline Erythema migrans, $\mathbf{n}(\%)$ & $23(16)$ & $19(25)$ & $37(36)$
\end{tabular}

Table 2. Age, gender and clinical characteristics of children with Lyme neuroborreliosis, on admission and at the 2-month follow-up in the two antibiotic treatment groups. 
Ceftriaxone i.v. Doxycycline p.o.

\begin{tabular}{|lrr}
\hline & $(\mathrm{n}=194)$ & $(\mathrm{n}=127)$ \\
\hline Age, median (range) & $6(1-18)$ & $10(4-19)$ \\
\hline Gender & & \\
\hline Female, $\mathrm{n}(\%)$ & $91(47)$ & $55(43)$ \\
\hline Male, $\mathrm{n}(\%)$ & $103(53)$ & $72(57)$ \\
\hline Major clinical characteristics on admission & & \\
\hline Facial nerve palsy, $\mathrm{n}(\%)$ & $122(63)$ & $89(70)$ \\
\hline Headache, $\mathrm{n}(\%)$ & $121(62)$ & $89(70)$ \\
\hline Fatigue, $\mathrm{n}(\%)$ & $139(72)$ & $84(66)$ \\
\hline Loss of appetite, $\mathrm{n}(\%)$ & $93(48)$ & $58(46)$ \\
\hline Fever, $\mathrm{n}(\%)$ & $83(43)$ & $55(43)$ \\
\hline Neck pain, $\mathrm{n}(\%)$ & $70(36)$ & $54(43)$ \\
\hline Neck stiffness, $\mathrm{n}(\%)$ & $43(22)$ & $42(33)$ \\
\hline Nausea, $\mathrm{n}(\%)$ & $40(21)$ & $35(28)$ \\
\hline Vertigo, $\mathrm{n}(\%)$ & $19(10)$ & $21(17)$ \\
\hline Pleocytosis in CSF, median (range) & & \\
\hline
\end{tabular}
Anti-Borrelia antibodies in CSF, n (\%)§
149 (77)
$80(63)$

\section{Diagnosis}

Definite LNB, n (\%)

$149(77)$

$80(63)$

Possible LNB, n (\%)

45 (23)

47 (37)

\section{Persistent signs and symptoms at follow-up*}

Facial nerve palsy, n (\%)

$26(13)$

$25(20)$

Headache, $\mathrm{n}(\%)$

$9(10)$

$5(4)$

Fatigue, n (\%)

5 (3)

$7(6)$

Abducens nerve palsy, $\mathrm{n}$ (\%)

1 (1)

$0(0)$

Affected balance, n (\%)

1 (1)

$0(0)$ 


\begin{tabular}{|c|c|c|}
\hline Hemiparesis, n (\%) & $1(1)$ & $0(0)$ \\
\hline Shoulder pain, n (\%) & $1(1)$ & $0(0)$ \\
\hline Joint pain, n (\%) & $0(0)$ & $1(1)$ \\
\hline Neck pain, n (\%) & $0(0)$ & $1(1)$ \\
\hline Radiating pain in extremity, n (\%) & $0(0)$ & $1(1)$ \\
\hline Hyperesthesia in extremity, n (\%) & $0(0)$ & $1(1)$ \\
\hline- & - & - \\
\hline Clinical outcome at follow-up & - & - \\
\hline Recovered, n (\%) & $155(80)$ & $94(74)$ \\
\hline Not recovered, n (\%) & $39(20)$ & $33(26)$ \\
\hline \multicolumn{3}{|l|}{ tients have reported several symptoms } \\
\hline \multicolumn{3}{|c|}{ sis: $>5 \times 10^{6} / \mathrm{L}$ white cells with a mononuclear cell dominance } \\
\hline \multicolumn{3}{|c|}{ al Borrelia specific antibody synthesis in CSF } \\
\hline \multicolumn{3}{|l|}{ me neuroborreliosis } \\
\hline
\end{tabular}

Table 3. Results of the logistic multivariate regression analysis for relevant clinical and laboratory data in association to clinical outcome at the 2-month follow-up (recovery/non-recovery). 
95\% Confidence

Interval

(Cl)

\begin{tabular}{llll} 
& p-value & $\begin{array}{l}\text { Odds ratio } \\
(\text { OR) }\end{array}$ \\
\hline Age & 0.547 & 1.03 & $(0.94-1.13)$ \\
\hline Gender, male & 0.490 & 1.22 & $(0.69-2.18)$ \\
\hline Known tick bite & 0.256 & 0.72 & $(0.41-1.27)$ \\
\hline Erythema migrans & 0.672 & 0.87 & $(0.45-1.68)$ \\
\hline Facial nerve palsy & $\mathbf{0 . 0 0 9}$ & $\mathbf{2 . 7 3}$ & $(1.29-5.78)$ \\
\hline Headache & 0.872 & 1.06 & $(0.54-2.08)$ \\
\hline Fatigue & 0.685 & 0.87 & $(0.44-1.70)$ \\
\hline Fever & $\mathbf{0 . 0 0 9}$ & $\mathbf{2 . 3 6}$ & $(1.24-4.50)$ \\
\hline Pleocytosis $\#$ & 0.951 & 1.00 & $(1.00-1.00)$ \\
\hline Anti-Borrelia-antibodies $\$$ & $\mathbf{0 . 0 0 8}$ & $\mathbf{0 . 4 1}$ & $(0.21-0.80)$ \\
\hline Antibiotic treatment, ceftriaxone & 0.887 & 1.05 & $(0.51-2.17)$
\end{tabular}

\# Pleocytosis: $>5 \times 10^{6} / \mathrm{L}$ white cells in CSF with a mononuclear cell dominance

$\S$ Intrathecal Borrelia specific antibody synthesis in CSF

CSF $=$ Cerebrospinal fluid

Figures 


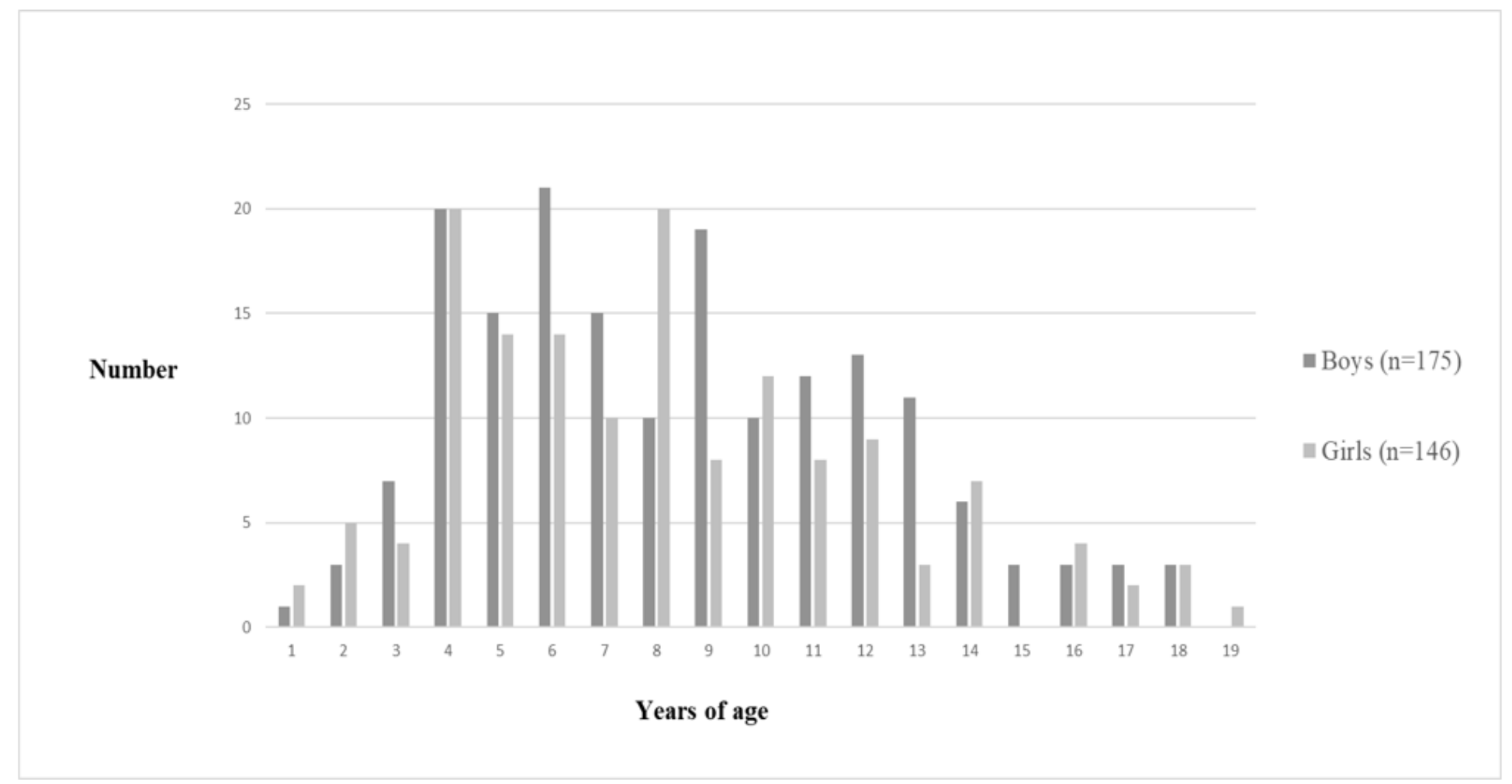

Figure 1

Distribution of age and gender among children with Lyme neuroborreliosis $(n=321)$.
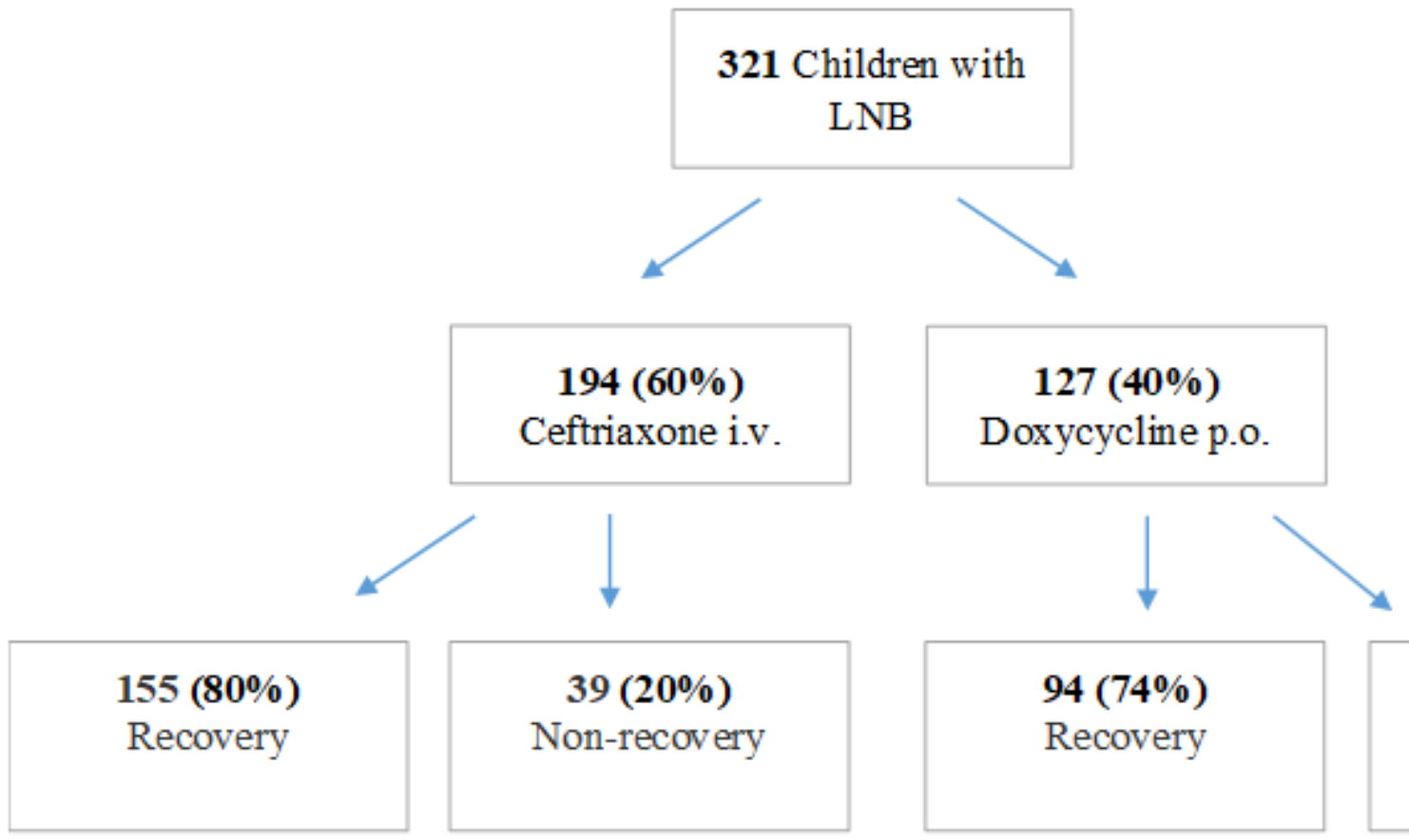

$33(26 \%)$

Non-recovery 
Figure 2

Flow chart showing different antibiotic treatment groups and clinical outcome as recovery/non-recovery in children with Lyme neuroboreliosis (LNB) at the 2-month follow-up. 